\title{
Field strength dependence of the high-frequency viscoelastic relaxation process in polyaniline/silicone oil electrorheological suspensions
}

\author{
P. Hiamtup ${ }^{1}$, A. Sirivat ${ }^{1 *}$, A. M. Jamieson ${ }^{2}$ \\ ${ }^{1}$ The Petroleum and Petrochemical College, Chulalongkorn University, Bangkok 10330, Thailand \\ ${ }^{2}$ Department of Macromolecular Science, Case Western Reserve University, Cleveland, Ohio 44106, USA
}

Received 15 July 2008; accepted in revised form 26 August 2008

\begin{abstract}
Polyaniline (PANI) was synthesized via oxidative coupling polymerization in acid conditions and de-doped in ammonia solution. The electrorheological (ER) properties of the PANI/silicone oil suspensions were investigated in the oscillatory mode shear, with particular focus on the high frequency region, where a crossover in $G^{\prime}(\omega)$ and $G^{\prime \prime}(\omega)$ signals the onset of a dissipative relaxation process, presumed to be associated with motion of PANI particles within the fibrillar structures generated by the electric field. The relationship between the crossover frequency, $\omega_{c}$, and the electric field strength $(E)$ was investigated as a function of matrix viscosity and shear strain. We find that $\omega_{c}$ increases with increasing electric field strength, and decreases with increases of matrix viscosity and strain amplitude. These observations are in qualitative agreement with a theoretical model, which relates the relaxation mechanism to the competition between hydrodynamic and electrostatic forces between PANI particles within thick fibrillar structures. At the crossover point, a critical scaling relation is found relating two dimensionless parameters, the Mason number $(\mathrm{Mn})$, and the Peclet number $(\mathrm{Pe})$, viz. $M n \sim(P e)^{0.09}$.
\end{abstract}

Keywords: rheology, electrorheological fluid, polyaniline, secondary crossover

\section{Introduction}

Electrorheological (ER) suspensions, first studied extensively by Winslow (1949), display dramatic changes in rheological properties upon application of an electric field [1]. A typical ER fluid is composed of micron-sized polarizable particles dispersed in a non-conducting medium. Under the action of the field, the particles form three dimensional fibrillar structures, which are aligned along the direction of the electric field and generate additional resistance against fluid motion [2]. With their adjustable viscosity, fast response, and reversible rheological behavior, ER fluids are regarded as smart materials, with potential for applications in active devices, which transform electric energy to mechanical energy [3]. Possible examples include clutches, breaks, shock absorbers, engine mounts, valves, flow pumps, and other variable control and servo devices [4].

A dimensionless parameter, the Mason number $(M n)$, has been widely used to describe the relative importance of hydrodynamic to electrostatic polarization forces, and has been shown to be useful to predict the ER suspension behavior in steady shear [5]. In the case of oscillatory shear, relatively few studies have explored the influence of the forces acting on the polarizable particles $[1,6]$. Of particular interest here are molecular dynamics simulation studies $[1,7]$ which found a frequency scaling of the dynamic shear moduli analogous to the

*Corresponding author, e-mail: anuvat_s@chula.ac.th

(c) BME-PT and GTE 
Mason number scaling of the steady shear response. Experimental measurements have reported the frequency scaling of the shear moduli of ER fluids based on conducting polymers consistent with the theoretical prediction $[8,9]$. In this paper, we focus on a prediction of molecular dynamics simulations that the oscillatory shear moduli of an ER fluid exhibit a high-frequency relaxation process traced to motion of the polarizable spheres within thick fibrillar clusters [1].

In previous studies, the use of polyaniline (PANI), and other semiconducting polymers, as the dispersed phase in an ER fluid has been extensively explored [8,10-12], since such polymers typically have a high intrinsic polarizability, even in the absence of any activator. PANI in particular has several advantages over other polymer particles such as low density, ease of synthesis and conductivity control, and thermal and environmental stability $[4,13]$. In the linear viscoelastic regime, a PANI suspension typically shows a liquid to solid transition at a critical electric field strength, whose value depends on particle concentration and host fluid viscosity [12]. Such behavior is consistent with the idea that there are two opposing forces which influence the assembly of the polarizable particles into fibrillar structures spanning the electrodes, viz. the electrostatic interparticle driving force for assembly, and the opposing hydrodynamic force associated with fluid motion [14].

In this study, we focus particularly on the fact that, at higher frequencies, the storage and loss shear moduli of PANI ER suspensions exhibit another crossover which signals the onset of a high-frequency relaxation mechanism presumed to be that predicted by molecular dynamics simulations [1]. We explore the effects of the medium viscosity, the shearing amplitude as well as the electric field strength on the crossover frequency, $\omega_{c}$, in the linear and nonlinear viscoelastic regimes. Dimensionless parameters, the Mason number $(M n)$ and Peclet number $(\mathrm{Pe})$, associated with the onset of the transition, are correlated according to a scaling relation.

\section{Experimental}

\subsection{Materials}

Aniline, $\mathrm{C}_{6} \mathrm{H}_{7} \mathrm{~N}$ (AR grade, Merck, Germany) was vacuum-distilled and used as the monomer. Ammonium peroxydisulphate, $\left(\mathrm{NH}_{4}\right)_{2} \mathrm{~S}_{2} \mathrm{O}_{8}$ (AR grade,
Merck, Germany) was used as the oxidant. $38 \%$ Hydrochloric acid, $\mathrm{HCl}$ (AR grade, Labscan, Thailand); $25 \%$ solution of ammonia, $\mathrm{NH}_{4} \mathrm{OH}$ (AR grade, Merck, Germany) and methanol, $\mathrm{CH}_{3} \mathrm{OH}$ (AR grade, Labscan, Germany) were used as received. The base fluids, silicone oil (AR grade, Dow Corning, USA) with density $0.96 \mathrm{~g} / \mathrm{cm}^{3}$ and kinematic viscosities of $10^{-4} \mathrm{~m}^{2} / \mathrm{s} \quad(100 \mathrm{cSt})$, $5 \cdot 10^{-4} \mathrm{~m}^{2} / \mathrm{s}$, and $10^{-3} \mathrm{~m}^{2} / \mathrm{s}$ were vacuum-dried and stored in a desiccator prior to use.

\subsection{Polymerization procedure}

PANI was synthesized via the oxidative coupling polymerization according to the method of Cao et al. (1989) [15]. After the course of polymerization, the precipitate was then dedoped by immersion in $3 \% \mathrm{NH}_{4} \mathrm{OH}$ in order to adjust its conductivity, before being vacuum dried and passed through a $38 \mu \mathrm{m}$ sieve shaker to control the particle size and its distribution.

To determine the electrical conductivity, PANI powder was pressed into disk pellets by a hydraulic press (diameter of $25 \mathrm{~mm}$ and $\sim 0.2 \mathrm{~mm}$ thick). Electrical conductivity was measured using a custom-built four-point probe at $30^{\circ} \mathrm{C}$. The measurements were performed in the linear Ohmic regime where the specific conductivity values are independent of the applied DC voltage.

\subsection{Preparation of ER fluids}

Prior to dispersion in silicone oil, PANI powder was dried for 2 days at room temperature to remove moisture in a vacuum oven at room temperature. The particles were then dispersed in the silicone oil with an ultrasonicator (Transonic 570/H, Elma, Singen, Germany) for 30 minutes at $25^{\circ} \mathrm{C}$. The PANI suspensions were then prepared at a volume fraction of 0.05 . The suspensions were stored in a dessiccator and redispersed by ultrasonication for a period of 10 minutes at $25^{\circ} \mathrm{C}$ before each experiment.

\subsection{Rheological measurements}

Dynamic rheological properties of the suspensions were investigated using a modified melt rheometer (ARES, Rheometric Scientific Inc., Piscataway, USA) with parallel plates (diameter of $50 \mathrm{~mm}$ ) 
attached via insulating spacers to the transducer and motor, with the gap size fixed at $0.2 \mathrm{~mm}$ throughout the experiment to avoid sloshing of samples at high frequency. A DC electric field was applied across the gap between the plates by a function generator (GFG-8216A, Instek, Melrose, USA) and a high voltage amplifier (Model 609E-6, Trek, USA). The electric field was applied for 10 minutes to obtain an equilibrium fibrillar or columnar structure before each measurement was taken. The experiments were carried out in the frequency sweep mode with frequency $(\omega)$ ranging from 0.1 to $100 \mathrm{rad} / \mathrm{s}$ at a temperature of $27 \pm 1^{\circ} \mathrm{C}$ to investigate the effect of electric field strength on $G^{\prime}$ and $G^{\prime \prime}$. Of particular interest was to correlate the applied field strength, $E$, with the $G^{\prime}-G^{\prime \prime}$ crossover frequency, $\omega_{c}$, as a function of medium viscosity and shear strain.

\section{Results and discussion}

\subsection{Electrorheological properties and $G^{\prime}-G^{\prime \prime}$ crossover of PANI/silicone oil suspensions}

The effect of the silicone oil viscosity and the strain amplitude, on the relationship between $\omega_{c}$ and $E$ was investigated for PANI suspensions prepared at the volume fraction of 0.05 in silicone oils of three different viscosities; 100, 500, $1000 \mathrm{cSt}$; the systems are coded as PANI/100, PANI/500, and PANI/1000, respectively. The PANI particle mean diameter, as determined by a particle size analyzer, is $23.5 \pm 2.37 \mu \mathrm{m}$; the gap/particle ratio is 8.5 . PANI particle electrical conductivity is $2.93 \cdot 10^{-9} \mathrm{~S} / \mathrm{cm}$. A PANI of low conductivity was chosen in order to be able to investigate ER behavior at high electric field strength without current leakage in the system [12].

The dynamic moduli, $G^{\prime}$ and $G^{\prime \prime}$, were measured in the frequency range 1-100 rad/s. In an ER system, in the absence of a field, the particles are randomly dispersed in suspension and the dynamic moduli generally exhibit liquid-like behavior such that $G^{\prime \prime}$ is significantly larger than $G^{\prime}$ over the entire frequency range. On application of a field, the particles form strings or columns spanning the gap between the electrodes, as a result of interfacial polarization due to migration of charge carriers in the PANI particle [16]. As the electric field strength increases to a critical value, the ER fluid passes through a series of transformations [12]. Two char-

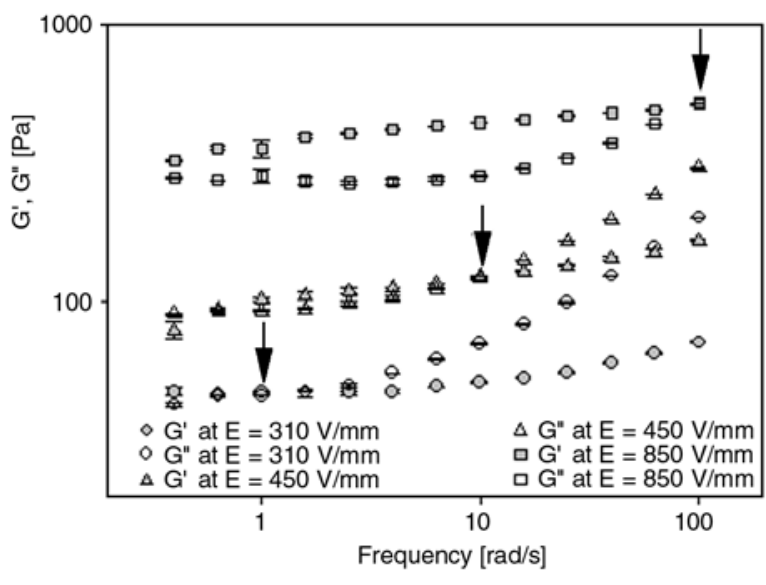

Figure 1. Frequency dependence of the shear moduli of the PANI/500 suspension at various electric field strengths, measured at a strain amplitude of $2 \%$, a temperature of $27 \pm 1^{\circ} \mathrm{C}$, and gap width $=$ $0.2 \mathrm{~mm}$.

acteristic transition points can be identified rheologically: the first is the sol-gel transition, characterized by the appearance of identical power law frequency scaling of the storage and loss moduli [17], and, subsequently, the liquid-to-solid transition associated with the crossover where $G^{\prime}$ becomes larger than $G^{\prime \prime}$, the latter coinciding with the visible appearance of fibrillar structures [12, 17].

Figure 1 shows the change in $G^{\prime}(\omega)$ and $G^{\prime \prime}(\omega)$ of the PANI/500 suspension as the applied field strength is increased. Evidently at the lowest deformation frequencies, $G^{\prime}$ is greater than $G^{\prime \prime}$, indicative that the polarization-induced fibrillar structures have formed, i.e. we are beyond the liquid-to-solid transition in this frequency range. Of more interest in this study is the fact that, at higher frequencies, indicated by arrows, we observe a second $G^{\prime}-G^{\prime \prime}$ crossover, where $G^{\prime \prime}$ becomes larger than $G^{\prime}$, characteristic of the onset of the high frequency relaxation process believed to be that identified in the molecular dynamics simulations [1]. It can be seen in Figure 1 that the crossover frequency, $\omega_{c}$, increases with electric field field strength, e.g. $E=$ $850 \mathrm{~V} / \mathrm{mm}$ for $\omega_{c}=100 \mathrm{rad} / \mathrm{s}$ as compared with $E=$ $310 \mathrm{~V} / \mathrm{mm}$ for $\omega_{c}=1 \mathrm{rad} / \mathrm{s}$.

\subsection{Effect of the host fluid viscosity and the strain amplitude}

Figure 2a shows the correlation between electric field strength and crossover frequency for various PANI/silicone oil suspensions having different host 

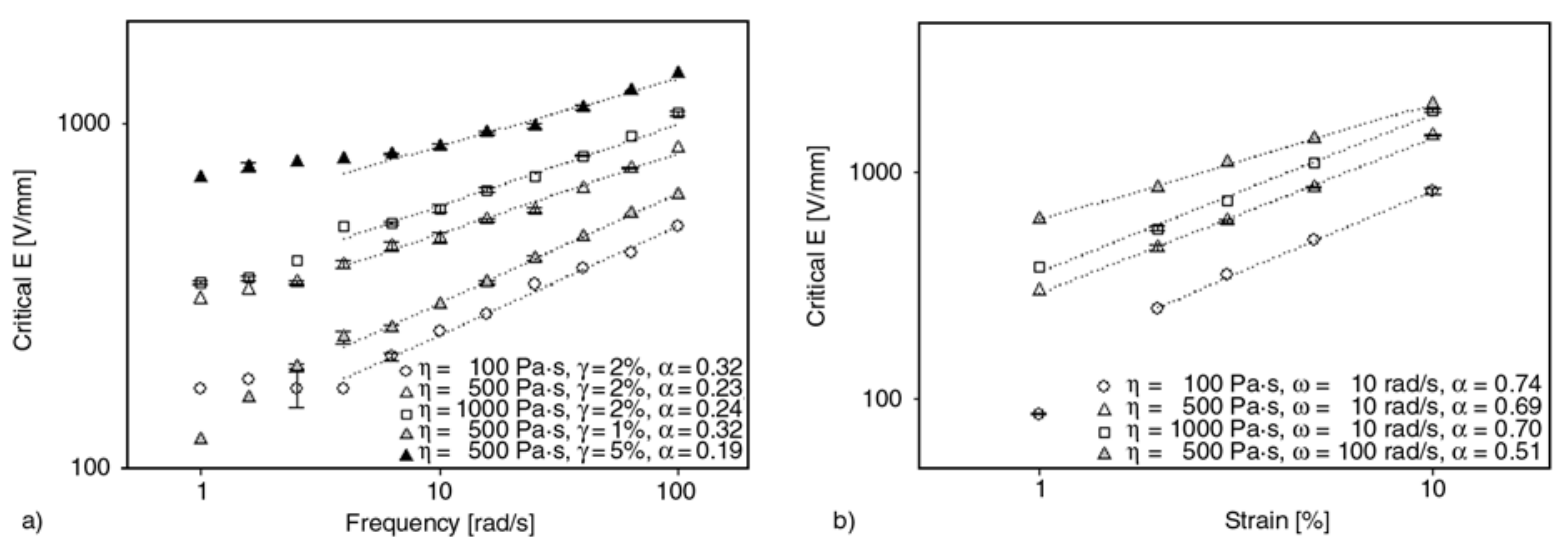

Figure 2. The dependence of critical electric field strength on: (a) frequency; and (b) strain amplitude for various PANI suspensions having different silicone oil viscosities, measured at temperature of $27 \pm 1^{\circ} \mathrm{C}$, gap $=0.2 \mathrm{~mm}$

viscosities. Included are data for PANI/100, PANI/ 500, and PANI/1000, all measured at a strain $\gamma=$ $2 \%$, as well as PANI/500 measured at $\gamma=1 \%$ and $\gamma=5 \%$. Figure 2 further illustrates that a higher electric field strength, $E$, correlates with a higher crossover frequency, $\omega_{c}$, and, in addition indicates that increased matrix viscosity leads to a higher electric field strength at a specified $\omega_{c}$. Figure $2 b$ indicates that increased strain amplitude leads to a higher electric field strength when $\omega_{c}$ is held constant. The correlation between $\log E$ and $\log \gamma$ at fixed crossover frequency is linear as shown in Figure $2 b$, which indicates a power law relationship. Figure $2 b$ further indicates that, at a fixed strain amplitude $E$ increases with increasing matrix viscosity at a fixed $\omega_{c}$.

These results are qualitatively consistent with the theoretical model that assumes the relaxation mechanism depends on the competition between hydrodynamic and polarization forces between particles within thick fibrillar columns [1]. Thus we may deduce that the onset of the relaxation process correlates with the condition where the two opposing forces, i.e. the shear force, $F_{\text {shear }}$, and the electrostatic force, $F_{\text {elec }}$, are in balance. In such a case we may use an argument proposed by Sakurai et al. [14] to relate the field strength to the crossover frequency, strain amplitude, matrix viscosity, and particle conductivity. Specifically, since, according to Equation (1):

$$
F_{\text {shear }}=6 \pi \eta_{m} \operatorname{\omega r}\left(\frac{d}{2}\right)^{2}
$$

where $d$ is the (assumed spherical) particle diameter, $\eta_{m}$ is the matrix viscosity and, according to Equation (2):

$$
F_{\text {elec }}=\frac{3 \pi p^{2}}{8 \varepsilon_{m} d^{4}}
$$

where $\varepsilon_{m}$ is the matrix dielectric constant and $p$ is the induced dipole moment, which, in the presence of a $d_{c}$ field may be written as Equation (3):

$$
p=\frac{\pi}{2} \varepsilon_{m}\left(\frac{d}{2}\right)^{3}\left(\frac{\sigma_{p}-\sigma_{m}}{\sigma_{p}+2 \sigma_{m}}\right) E=\frac{\pi}{2} \varepsilon_{m}\left(\frac{d}{2}\right)^{3} \beta E
$$

where $\sigma_{p}$ and $\sigma_{m}$ are the electrical conductivities of PANI particles and matrix, respectively. Equating Equations (1) and (2) we obtained Equation (4) [14]:

$E_{c r i t}=\frac{32}{\pi} \sqrt{\frac{\omega_{c} \gamma \eta_{m}}{\varepsilon_{m} \beta^{2}}}$

where $\omega_{c}$ is the crossover frequency, $\gamma$ is the strain amplitude, $\eta_{m}$ is the matrix viscosity, and $\varepsilon_{m}$ is the dielectric constant of the matrix. Thus, Equation (4) predicts that $E_{c r i t} \alpha \omega_{c}^{1 / 2}$ at fixed $\gamma$ and $\eta_{m}$, and $E_{c} \alpha \gamma^{1 / 2}$ at fixed $\omega_{c}$ and $\eta_{m}$. In comparison, the experimental data in Figures $2 \mathrm{a}$ and $2 \mathrm{~b}$ indicate that indeed $E_{c} \alpha \omega_{c}^{\alpha}$ and $E_{c} \alpha \gamma^{\beta}$, but $\alpha$ varies between $0.19-0.32$, and $\beta$ varies between $0.51-0.74$ amongst various host fluid viscosities tested. The origin of the discrepancies is not clear. One possibility relates to the fact that Equation (4) pertains to a suspension of monodisperse spheres, whereas the PANI suspension has particles of irregular shapes and a broad particle size distribution. Also, Equa- 
tion (4) considers only pairwise interactions between particles, and ignores multi-particle interactions [12]. A potential complication in the case of the dependence on shear strain is that the experiments extend from the linear to the nonlinear viscoelastic regime. Another factor is that the theoretical model, based on an order of magnitude analysis, assumes that the two primary forces are proportional to each other; in experiments such ideal condition rarely exists due to other secondary forces that may become involved as $P e$ number is varied [7].

\subsection{Dimensionless group analysis}

Under action of the electric field, an ER suspension experiences several inter-particle forces; the electrostatic force, the Brownian force, the short-range repulsion, the hydrodynamic interaction, as well as other colloidal forces. Dimensionless groups arising from such forces are commonly used to describe and interpret suspension behavior in terms of the relative importance of competing contributions [7].

For steady shear flow, the relative importance of the viscous to the electrostatic polarization forces is described by the Mason number [5], defined by Equation (5):

$$
M n=\frac{\eta_{m} \gamma}{2 \varepsilon_{0} \varepsilon_{m} \beta^{2} E^{2}}
$$

The relative importance of viscous to thermal forces is described by the Peclet number [18], defined by Equation (6):

$$
P e=\frac{3 \pi\left(\frac{d}{2}\right)^{2} \eta_{m} \gamma}{k_{B} T}
$$

where $\eta_{m}$ is the matrix viscosity, $\gamma$ is the shear rate, $\varepsilon_{0}$ is the electric permittivity of vacuum, $\varepsilon_{m}$ is the matrix dielectric constant, $T$ is the absolute temperature, and $k_{B}$ is the Boltzmann constant.

In order to make Equations (5) and (6) applicable for an oscillatory shear flow of PANI suspensions, we replace $\eta_{m}$ the steady shear matrix viscosity by $\eta_{s}$ the steady shear suspension viscosity $\left(\eta_{s} \geq \eta_{m}\right)$, and finally the steady shear suspension viscosity is replaced by the dynamic suspension viscosity, viz.
$\eta_{s}^{\prime}(\omega)=G_{s}^{\prime \prime}(\omega) / \omega$ and $\gamma=\omega \gamma$. Thus, Equation (5) becomes Equation (7):

$$
M n=\frac{G_{s}^{\prime \prime}(\omega) \gamma}{2 \varepsilon_{0} \varepsilon_{m} \beta^{2} E^{2}}
$$

and Equation (6) becomes Equation (8):

$P e=\frac{3 \pi\left(\frac{d}{2}\right)^{3} G_{s}^{\prime \prime}(\omega) \gamma}{k_{B} T}$

To calculate $M n$ and $P e$, we used $\varepsilon_{0}=8.85 \mathrm{pF} / \mathrm{m}$ and $\varepsilon_{m}=2.4$ as the electric permittivity of vacuum and the matrix dielectric constant, respectively. The relative conductivity, $\beta$, is approximately equal to 1 as $\sigma_{m}=7.7 \cdot 10^{-15} \mathrm{~S} / \mathrm{cm} \mathrm{[19]} \mathrm{and} \sigma_{p}=2.93 \cdot 10^{-9} \mathrm{~S} / \mathrm{cm}$ are the conductivity values of matrix and particle, respectively. $k_{B}=1.38 \cdot 10^{-23} \mathrm{~J} \cdot \mathrm{K}^{-1}$ is the Boltzmann constant, and $T=300 \mathrm{~K}$ is the absolute temperature. $d=23.5 \mu \mathrm{m}$ is the average particle diameter.

Figure 3 shows a plot of the dimensionless parameter $M n$ versus $P e$, at the crossover frequency for the $\mathrm{PANI} /$ silicone oil suspensions at various viscosities, shear frequencies and strain amplitudes. It is evident that the data points for all systems approximately collapse into a single function of the dimensionless parameter $P e$, which corresponds to a least squares scaling relation of the form $M n=$ $0.0605(P e)^{0.09}$.

Several different definitions of the Mason number can also be used, and we also tested the scaling relation between $\mathrm{Mn}$ and $\mathrm{Pe}$ using the Sakurai definition of $M n$, based on Equations (1) and (2) for $F_{\text {elec }}$ and $F_{\text {shear }}$, respectively, viz. Equation (9):

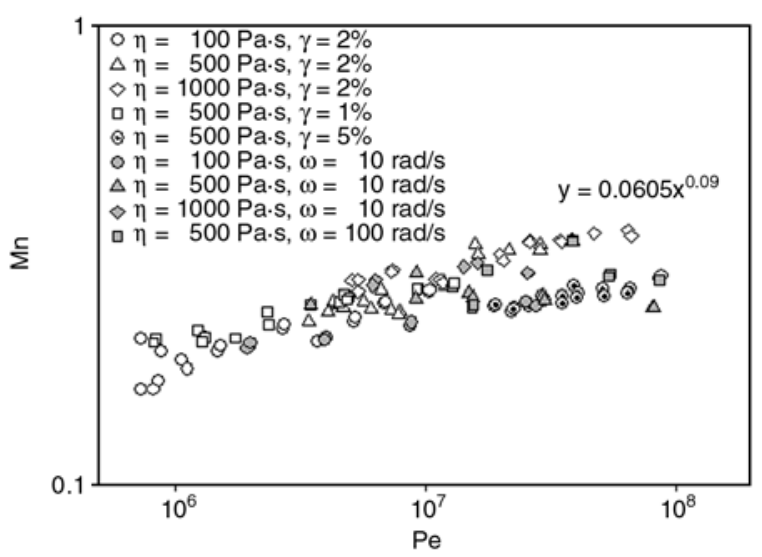

Figure 3. The dimensionless parameter $M n$ versus $P e$ for PANI suspensions having different silicone oil viscosities. 
$M n_{\text {Sakurai }}=\frac{4 d^{6} \varepsilon_{m} G_{s}^{\prime \prime}(\omega) \gamma_{0}}{p^{2}}$

where $p$ is the dipole moment induced by MaxwellWagner type-polarization, given by Equation (3). Combining Equation (2) for $F_{\text {shear }}$ with an expression for the thermal force $F_{\text {thermal }}=k_{B} T /(d / 2)$ [5], one obtains Equation (10), an expression for the Peclet number slightly different from Equations (8):

$$
P e_{\text {Sakurai }}=\frac{3 \pi d^{3} G_{s}^{\prime \prime}(\omega) \gamma}{4 k_{B} T}
$$

The corresponding plot of $M n_{\text {Sakurai }}$ versus Pe Sakurai is shown in Figure 4, and leads to a scaling relation of $M n=6,79(P e)^{0,09}$.

In discussing the results in Figures 3 and 4, we note, first, that, since $\mathrm{Pe}$ is of the order $10^{6}-10^{9}$, and $M n$ of the order $0.1-1.0$ or $10-10^{2}$, depending on which definition is used, it is clear that the magnitude of the Brownian force is relatively small in comparison to the shear and electrostatic forces. Also, the scaling exponent 0.09 indicates that the Mason number, as defined in either Equation (7) or Equation (9), is not quite a universal constant at the crossover frequency. Specifically, bearing in mind that only isothermal data were obtained in these experiments, Figures 3 and 4, indicate, over the range of conditions studied, $M n$ increases by about $30 \%$ whereas the shear force increases by over two decades. The dependence of Mason number on $P e$ number can be interpreted as follows: as the viscous shear force is increased, or $P e$ number is

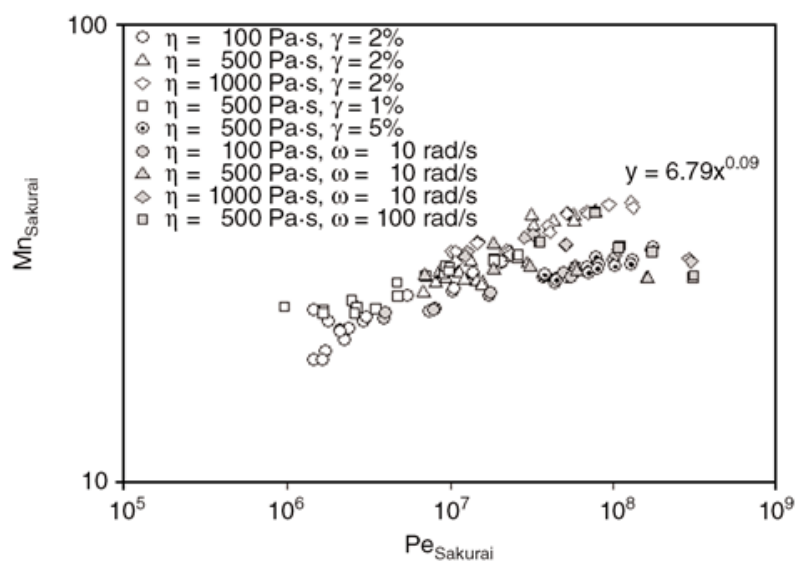

Figure 4. The dimensionless parameter $M n_{\text {Sakurai }}$ versus Pesakurai for PANI suspensions having different silicone oil viscosities. increased, the electrostatic force required to keep the fibrillar structures from rupture becomes proportionally less. The argument can be also cast in terms of the Brownian force which becomes relatively smaller as $P e$ number is increased; thus a smaller magnitude of the electrostatic force is required to keep the structure intact.

\section{Conclusions}

Polyaniline (PANI) was synthesized via the oxidative coupling polymerization in acid conditions and de-doped in $3 \% \mathrm{NH}_{4} \mathrm{OH}$. The ER properties of $\mathrm{PANI} /$ silicone oil suspensions were investigated with a particular focus on the crossover in the storage and loss shear moduli which signals the onset of a high frequency relaxation process associated with the motion of PANI particles within the fibrillar columns produced by the applied field. The relationship between the electric field strength $(E)$ and the deformation frequency $\left(\omega_{c}\right)$ at the crossover point was investigated as a function of medium viscosity and shearing amplitude. Qualitatively consistent with a theoretical model, which relates the relaxation process to competition of hydrodynamic and electrostatic forces between polarizable particles within the fibrillar columns, the value of $E_{c}$ increases with increase in each of these three system variables. Quantitative deviations from the theory are found however, manifested as deviations in the scaling exponent of the dependences of $E_{c}$ on shearing frequency and strain amplitude from the predicted values of 0.5 . The dimensionless parameters, Mason number $(\mathrm{Mn})$ and Peclet number $(\mathrm{Pe})$, of the suspension at the critical point were also studied. Data plotted as $M n$ versus $P e$, for all systems approximately collapse into a single function of the form $M n=0.0605(P e)^{0.09}$. This indicates the Mason number is not a universal constant at the crossover point, but that there is an approximate universal scaling between the viscous stress and the electrostatic polarization stress.

\section{Acknowledgements}

The authors would like to acknowledge the financial supports provided by The Thailand Research Fund (TRF), RGJ grant no. PHD/0234/2544 and BRG; the Conductive \& Electroactive Polymers Research Unit of Chulalongkorn University; the Royal Thai Government (Budget of Fiscal Year 2551); and the Petroleum Petrochemical and 
Advanced Materials Consortium. AMJ would like to acknowledge the support from the US National Science Foundation.

\section{References}

[1] Klingenberg D. J.: Simulation of the dynamic oscillatory response of electrorheological suspensions: Demonstration of a relaxation mechanism. Journal of Rheology, 37, 199-214 (1993).

[2] Tao R., Sun J. M.: Three-dimensional structure of induced electrorheological solid. Physical Review Letters, 67, 398-401 (1991).

[3] Choi H. J., Cho M. S., To K.: Electrorheological characterization of polyaniline dispersions. Physica A, 254, 272-279 (1998).

[4] Jang W. H., Kim J. W., Choi H. J., Jhon M. S.: Synthesis and electrorheology of camphorsulfonic acid doped polyaniline suspensions. Colloid and Polymer Science, 279, 823-827 (2001).

[5] Marshall L., Zukoski IV C. F., Goodwin J. W.: Effects of electric fields on the rheology of nonaqueous concentrated suspensions. Journal of the Chemical Society, Faraday Transactions 1: Physical Chemistry in Condensed Phases, 85, 2785-2795 (1989).

[6] Toor W.: Structure formation in electrorheological fluids. Journal of Colloid and Interface Science, 156, 335-339 (1993).

[7] Parthasarathy M., Klingenberg D. J.: Electrorheology: Mechanisms and models. Materials Science and Engineering: R: Reports, 17, 57-103 (1996).

[8] Cho M. S., Lee J. H., Choi H. J., Ahn K. H., Lee S. J., Jeon D.: Linear viscoelasticity of semiconducting polyaniline based electrorheological suspensions. Journal of Materials Science, 39, 1377-1382 (2004).

[9] Chotpattananont D., Sirivat A., Jamieson A. M.: Electrorheological properties of perchloric acid-doped polythiophene suspensions. Colloid and Polymer Science, 282, 357-365 (2004).
[10] Kim J. W., Choi H. J., Lee H. G.: Damping characteristics of polyaniline-based electrorheological fluid. Journal of Industrial and Engineering Chemistry, 7, 218-222 (2001).

[11] Lin D. D., Zhang Z. J., Zhao B. Y., Chen L. S., Hu K.: Rapid synthesis of porous polyaniline and its application in electrorheological fluid. Smart Materials and Structures, 15, 1641-1645 (2006).

[12] Hiamtup P. A., Sirivat A., Jamieson A. M.: Electrorheological properties of polyaniline suspensions: Field-induced liquid to solid transition and residual gel structure. Journal of Colloid and Interface Science, 295, 270-278 (2006).

[13] Pinto N. J., Acosta A. A., Sinha G. P., Aliev F. M.: Dielectric permittivity study on weakly doped conducting polymers based on polyaniline and its derivatives. Synthetic Metals, 113, 77-81 (2000).

[14] Sakurai R., See H., Saito T., Sumita M.: Effect of matrix viscoelasticity on the electrorheological properties of particle suspensions. Journal of Non-Newtonian Fluid Mechanics, 81, 235-250 (1999).

[15] Cao Y., Andreatta A., Heeger A. J., Smith P.: Influence of chemical polymerization conditions on the properties of polyaniline. Polymer, 30, 2305-2311 (1989).

[16] Block H., Kelly J. P., Qin A., Watson T.: Materials and mechanisms in electrorheology. Langmuir, 6, 6-14 (1990).

[17] Chin B. D., Winter H. H.: Field-induced gelation, yield stress, and fragility of an electro-rheological suspension. Rheologica Acta, 41, 265-275 (2002).

[18] Melrose J. R.: Brownian dynamics simulation of dipole suspensions under shear: The phase diagram. Molecular Physics, 76, 635-660 (1992).

[19] Rejon L., Manero O., Lira-Galeana C.: Rheological, dielectric and structural characterization of asphaltene suspensions under DC electric fields. Fuel, 83, 471-476 (2004). 